\title{
Evaporating very small grains as tracers of the UV radiation field in photo-dissociation regions
}

\author{
P. Pilleri ${ }^{1,2,3,4}$, J. Montillaud ${ }^{1,2}$, O. Berné ${ }^{1,2}$, and C. Joblin ${ }^{1,2}$ \\ 1 Université de Toulouse, UPS-OMP, IRAP, Toulouse, France \\ e-mail: p.pilleri@oan.es \\ 2 CNRS, IRAP, 9 Av. colonel Roche, BP 44346, 31028 Toulouse Cedex 4, France \\ 3 Centro de Astrobiología (INTA-CSIC), Ctra. M-108, km. 4, 28850 Torrejón de Ardoz, Spain \\ 4 Observatorio Astronómico Nacional, Apdo. 112, 28803 Alcalá de Henares, Spain
}

Received 12 October 2010 / Accepted 19 April 2012

\section{ABSTRACT}

\begin{abstract}
Context. In photo-dissociation regions (PDRs), polycyclic aromatic hydrocarbons (PAHs) may be produced by evaporation of very small grains (VSGs) by the impinging UV radiation field from a nearby star.

Aims. We quantitatively investigate the transition zone between evaporating VSGs (eVSGs) and PAHs in several PDRs.

Methods. We studied the relative contribution of PAHs and eVSGs to the mid-IR emission in a wide range of excitation conditions. We fitted the observed mid-IR emission of PDRs by using a set of template band emission spectra of PAHs, eVSGs, and gas lines. The fitting tool PAHTAT (PAH Toulouse Astronomical Templates) is made available to the community as an IDL routine. From the results of the fit, we derived the fraction of carbon $f_{\mathrm{eVSG}}$ locked in eVSGs and compared it to the intensity of the local UV radiation field.

Results. We show a clear decrease of $f_{\text {evsG }}$ with increasing intensity of the local UV radiation field, which supports the scenario of photo-destruction of eVSGs. Conversely, this dependence can be used to quantify the intensity of the UV radiation field for different PDRs, including unresolved ones.

Conclusions. PAHTAT can be used to trace the intensity of the local UV radiation field in regions where eVSGs evaporate, which correspond to relatively dense $\left(n_{\mathrm{H}}=\left[100,10^{5}\right] \mathrm{cm}^{-3}\right)$ and UV irradiated PDRs $\left(G_{0}=\left[100,5 \times 10^{4}\right]\right)$ where $\mathrm{H}_{2}$ emits in rotational lines.
\end{abstract}

Key words. photon-dominated region (PDR) - astrochemistry - dust, extinction - ISM: lines and bands - infrared: ISM ISM: molecules

\section{Introduction}

The mid-infrared (mid-IR) spectrum of photo-dissociation regions (PDRs) is dominated by the aromatic infrared bands (AIBs) attributed to the emission of polycyclic aromatic hydrocarbons (PAHs, Léger \& Puget 1984; Allamandola et al. 1985; Joblin \& Tielens 2011). Very small grains (VSGs) are another class of mid-IR emitters if they are small enough to be stochastically heated (Désert et al. 1990). The VSGs are generally considered to be carbonaceous (Désert et al. 1986) but Li \& Draine (2001b) concluded that the presence of very small silicate grains is not excluded and can involve up to $10 \%$ of interstellar $\mathrm{Si}$. Cesarsky et al. (2000) suggested a link between PAHs and VSGs, in which the latter are processed by the UV radiation field or by shocks giving birth to free PAHs.

Using blind-source separation methods, Rapacioli et al. (2005) and Berné et al. (2007) extracted three independent spectra from the mid-IR emission, two molecular PAH-type spectra attributed to neutral and ionised PAH populations, respectively, and one consisting of continuum and broad band emissions, which was attributed to some VSGs. The relative spatial distribution between PAHs and these VSGs additionally supported the idea that free flying PAH molecules are produced by photo-evaporation of VSGs under UV irradiation at the border of PDRs (Rapacioli et al. 2005; Berné et al. 2007). Although they are called VSGs, this population is not strictly that included in the Désert et al. (1990) model: it is better described by the PAH grains of Li \& Draine (2001a). In the following, we will refer to them as evaporating VSG (eVSGs).

Using the template spectra derived by Rapacioli et al. (2005) and Berné et al. (2007), Joblin et al. (2008) and Berné et al. (2009b) were able to fit the mid-IR spectra of objects in different evolutionary stages, such as planetary nebulae and protoplanetary disks. A spectrum attributed to large ionised PAHs $\left(\mathrm{PAH}^{x}\right)$ was introduced to fit highly irradiated objects. In Berné et al. (2009b) and Berné et al. (2009a), the relative intensities of the different mid-IR components were related to physical quantities such as the spectral type of the central illuminating star for proto-planetary disks, or the ionisation parameters and radiation field for the Monoceros R2 compact HII region. While it is clear, in all the above studies, that the evolution of eVSGs into PAHs is related to the intensity of the UV field, this has never been quantified.

In this paper, we search for a quantitative link between the intensity of the UV radiation field and the chemical evolution of the AIB carriers in terms of processing of eVSGs into PAHs. Section 2 describes the PDR sample. Section 3 presents the fitting tool we have developed to decompose mid-IR spectra into chemical populations, which takes into account dust extinction. We apply this method to our PDR sample and present the results in Sect. 4. Section 5 describes the relation between the local physical conditions and the evolution of the AIB carriers 
A\&A 542, A69 (2012)

Table 1. Observational parameters for PDRs with spatially resolved mid-IR populations.

\begin{tabular}{|c|c|c|c|c|c|c|c|c|}
\hline Object & Star & $\begin{array}{l}\text { Distance } \\
{[\mathrm{pc}]^{a}}\end{array}$ & $\begin{array}{l}\text { Spectral } \\
\text { type }\end{array}$ & $\begin{array}{c}\text { Kurucz } \\
\text { spectrum* }[\mathrm{K}]\end{array}$ & $\begin{array}{c}\text { Radius* }^{*} \\
{\left[R_{\odot}\right]}\end{array}$ & $\begin{array}{c}d_{\text {front }} \\
{\left[{ }^{\prime \prime}\right]}\end{array}$ & $G_{0}^{\text {front }}$ & Instrument \\
\hline $\begin{array}{l}\text { NGC } 7023 \mathrm{NW} \\
\text { NGC } 7023 \mathrm{~S} \\
\text { NGC } 7023 \mathrm{E}\end{array}$ & HD 200775 & 430 & $\mathrm{~B} 3 \mathrm{Ve}-\mathrm{B} 5^{b, c, d}$ & $2 \times 15000^{e}$ & 10 & $\begin{array}{c}42 \\
55 \\
155 \\
\end{array}$ & $\begin{array}{c}2600 \\
1500 \\
250 \\
\end{array}$ & $\begin{array}{l}\text { Spitzer IRS-SL } \\
\text { ISO-CAM cvf } \\
\text { ISO-CAM cvf }\end{array}$ \\
\hline $\begin{array}{l}\text { NGC } 2023 \mathrm{~N} \\
\text { NGC } 2023 \mathrm{~S}\end{array}$ & HD 37903 & 470 & $\mathrm{~B} 1.5 \mathrm{~V}^{f}$ & 24000 & 6 & $\begin{array}{l}164 \\
67\end{array}$ & $\begin{array}{c}400 \\
4000\end{array}$ & $\begin{array}{l}\text { Spitzer IRS-SL } \\
\text { Spitzer IRS-SL }\end{array}$ \\
\hline$\rho$-Oph filament & HD 147889 & 118 & B2III-B2V & 22000 & 5 & 610 & 520 & ISO-CAM cvf \\
\hline
\end{tabular}

Notes. ${ }^{(*)}$ Derived from spectral type.

References. ${ }^{(a)}$ van den Ancker et al. (1997); ${ }^{(b)}$ Racine (1968); ${ }^{(c)}$ Finkenzeller (1985); ${ }^{(d)}$ Witt et al. (2006); ${ }^{(e)}$ Alecian et al. $(2008) ; ~\left({ }^{(f)}\right.$ Diplas \& Savage (1994).

and illustrates the potential of our method for characterising the UV field in a variety of PDR environments.

\section{The PDR sample}

We analysed mid-IR observations of several PDRs that span a wide range of UV irradiation conditions. The majority of the observations discussed here were obtained with the short-low (SL) module of the Infrared Spectrograph (IRS) (Houck et al. 2004) onboard Spitzer (Werner et al. 2004) in spectral mapping mode. Data reduction was performed with the CUBISM software (Smith et al. 2007a) and consisted of cube assembling, calibration, flux correction for extended sources and bad pixel removal. For the objects that were not observed with IRS, we used ISOCAM data available in the ISO data archive as highly processed data products (Boulanger et al. 2005).

\subsection{PDRs with spatially resolved mid-IR populations}

Our sample contains several PDRs (Table 1) where the emission from the PAH and eVSG populations (see Sect. 3) can be spatially isolated. These PDRs are referred to as PDRs with spatially resolved mid-IR populations. It is possible to study the variations of the mid-IR spectral properties in these sources (Sect. 3) and link them to the local physical properties such as the local density and UV field (Sect. 5) as a function of the depth inside the PDR. To estimate the intensity of the UV radiation field at the PDR front, we used modelled stellar spectra from Kurucz (1993) and applied a dilution factor based on their projected distance to the PDR front. All parameters used to calculate the intensity of the UV radiation field at the PDR fronts are reported in Table 1. In the following, we will express the UV field intensity $G_{0}$ in terms of the Habing field, which corresponds to $1.6 \times 10^{-3} \mathrm{erg} \mathrm{cm}^{-2} \mathrm{~s}^{-1}$ when integrated between 91.2 and $240 \mathrm{~nm}$ (Habing 1968). The geometrical model assumed for these PDRs is discussed in Appendix A.2.

$\rho$-oph filament. Assuming typical spectral properties for the B2 star HD 147889 and using geometrical dilution to the projected distance of $0.4 \mathrm{pc}$ between the star and the PDR leads to an intensity of the UV radiation field of $G_{0} \sim 520$.

NGC 2023. For NGC 2023 an extinction of $A_{\mathrm{V}}=1.1$ mag was applied, reflecting absorbing dust around the illuminating star HD 37903 (Compiègne et al. 2008). Using a distance of 0.38 and $0.13 \mathrm{pc}$ for the north and south PDRs, respectively, yields an estimate of the UV radiation field intensity of $G_{0} \sim 400$ and $G_{0} \sim 4000$. These estimates are consistent with previous values obtained in the far-IR study by Burton et al. (1998).

NGC 7023. The estimate of the UV radiation field intensity at the PDR front in NGC $7023 \mathrm{NW}$ is more uncertain. Chokshi et al. (1988) estimated $G_{0} \sim 2.4 \times 10^{3}$ through far-IR observations and modelling, but values as high as $G_{0} \sim 10^{4}$ have been proposed to explain the observed $\mathrm{H}_{2}$ ortho-to-para ratio (Fuente et al. 1999) towards this PDR. This value agrees with the value determined by assuming typical stellar properties for the corresponding spectral type of the illuminating binary star (Alecian et al. 2008) and geometrical dilution to the PDR front. However, extinction between the star surface and the PDR may attenuate the radiation field, yielding a lower value at the PDR front. In this work, an extinction correction of $A_{\mathrm{V}}=1.5$ was derived from the IUE spectrum measured on the star and was applied on the corresponding Kurucz spectrum. Assuming that the same extinction factor applies between the star and the PDR leads to an estimate for the NW PDR of $G_{0} \sim 2.6 \times 10^{3}$, which is consistent with the value of Chokshi et al. (1988).

\subsection{PDRs with mixed mid-IR populations}

In the previous section we have defined the PDRs with spatially resolved mid-IR populations as those where the PAHs and eVSGs can be spatially isolated. The PDRs of our sample that do not follow this criterion are referred to as PDRs with mixed mid-IR populations. The fact that in these sources the emission from PAHs and eVSGs cannot be isolated may result from different effects, e.g. from the small spatial extent of the PDR, a lower signal-to-noise ratio in the data, specific geometrical properties, etc. For each of these sources we analysed an averaged spectrum towards the peak of the mid-IR emission. The sample of PDRs with mixed mid-IR populations comprises a wide range of irradiation conditions, from $G_{0} \sim 100$ for the Horsehead Nebula to $G_{0} \sim 4 \times 10^{4}$ for the Orion Bar. For these well-studied objects, a reliable estimate of the intensity of the UV radiation field can be found in the literature.

\section{A spectral fitting tool for studying the chemical evolution of AIB carriers: PAHTAT}

Our motivation here is to provide a tool that allows us to decompose observed mid-IR $(5.5-14 \mu \mathrm{m})$ spectra into a limited set of components that can provide information on the chemical nature of the emitters. We note that there already exists an AIB fitting tool called PAHFIT, proposed by Smith et al. (2007b), which 
P. Pilleri et al.: Evaporating very small grains as tracers of the UV field in PDRs

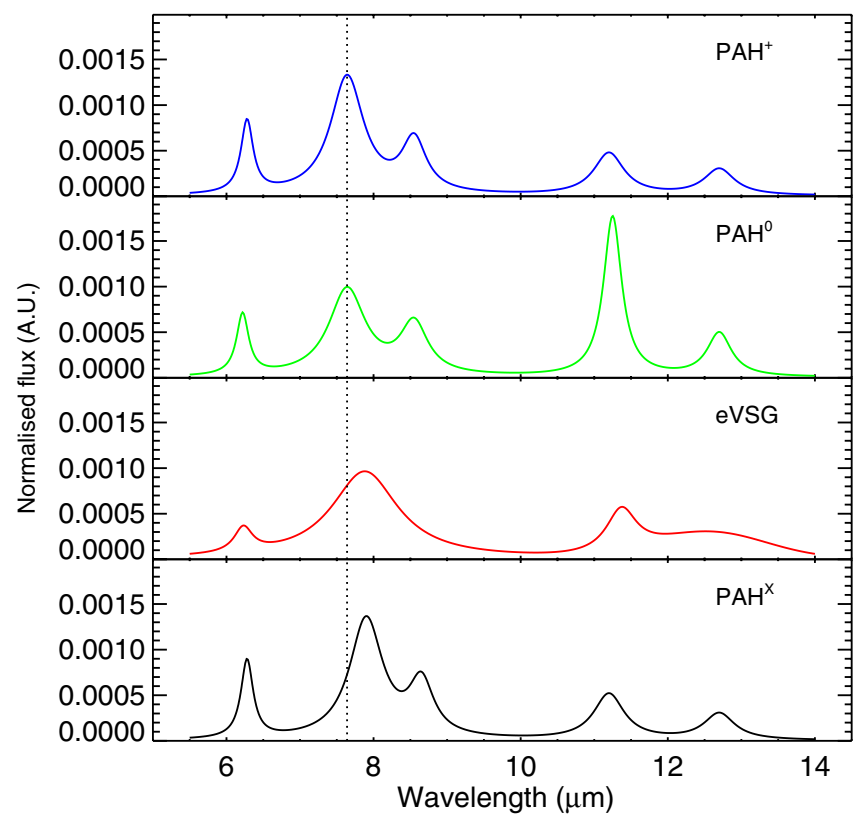

Fig. 1. Template spectra used in the fitting procedure. The eVSG spectrum from Joblin et al. (2008) was slightly improved to better fit the observed spectrum by adding a plateau in the $12-14 \mu \mathrm{m}$ range. Spectra were normalised to their integrated intensity between 5.5 and $14 \mu \mathrm{m}$.

decomposes mid-IR spectra using a collection of lorentzians and black body functions with a large number of parameters. While PAHFIT provides high-quality fits that are useful e.g. to extract the integrated intensity from individual bands, our aim is to decompose the observations using band template spectra for PAHs and eVSGs, minor additional unattributed PAH features, gas lines, and a function to describe the continuum ${ }^{1}$.

\subsection{Band emission}

Applying blind-signal separation methods to ISO and Spitzer spectro-imagery data of several PDRs Rapacioli et al. (2005) and Berné et al. (2007) extracted from the observations a set of three elementary mid-IR spectra that can reproduce the AIB emission. Joblin et al. (2008) and Berné et al. (2009b) found that a fourth spectral component was needed to explain the observed spectra in highly irradiated environments. PAHTAT is based on these four astronomical template band spectra (Fig. 1). Here, we briefly present the spectral properties of this set of templates and their chemical assignment. Details on the spectral characteristics of the templates (e.g. relative band strength, position, width, etc.), how they were obtained as well as the populations they represent can be found in Joblin et al. (2008) ${ }^{2}$. The first component is the neutral $\mathrm{PAH}\left(\mathrm{PAH}^{0}\right)$ spectrum, which is mainly characterised by a high $\mathrm{C}-\mathrm{H}(11.2 \mu \mathrm{m})$ over $\mathrm{C}-\mathrm{C}$ $(7.7 \mu \mathrm{m})$ band ratio. The second component is the PAH cation $\left(\mathrm{PAH}^{+}\right)$spectrum, which displays on the contrary a low $11.2 \mu \mathrm{m}$ over $7.7 \mu \mathrm{m}$ band ratio. The third PAH component is associated to large ( $\sim 100 \mathrm{C}$ atoms $)$ ionised PAHs, referred to as $\mathrm{PAH}^{x}$, whereas the $\mathrm{PAH}^{+}$and $\mathrm{PAH}^{0}$ populations would be dominated

\footnotetext{
1 The corresponding IDL code PAHTAT (PAH Toulouse Astronomical Templates) is available for download at http://userpages .irap.omp.eu/ cjoblin/PAHTAT

2 These templates can be downloaded on the PAHTAT webpage at http://userpages.irap.omp. eu/ cjoblin/PAHTAT
}

by smaller species. Its spectrum shares the same characteristics as that of $\mathrm{PAH}^{+}$but has a "7.7" $\mu \mathrm{m}$ band that is shifted by $0.15 \mu \mathrm{m}$ to the red compared to $\mathrm{PAH}^{+}$(for a discussion of the reason for this shift, see Joblin et al. 2008). The fourth component is the eVSG spectrum, whose carriers are still discussed and have typical sizes of $~ 500 \mathrm{C}$ atoms (Rapacioli et al. 2005). PAH clusters (Rapacioli et al. 2006) or complexes of PAHs with Fe atoms (Simon \& Joblin 2009) have been proposed. eVSGs are thought to carry continuum emission, but here we have treated band and continuum emission separately. The eVSG band spectrum is made of broad features at 7.7 and $11.2 \mu \mathrm{m}$ and has no $8.6 \mu \mathrm{m}$ band (Fig. 1). We improved this spectrum as compared to Joblin et al. (2008) by adding a Gaussian plateau centred at $12.6 \mu \mathrm{m}$ with a $F W H M$ of $1.76 \mu \mathrm{m}$ and a relative intensity compared to the $7.7 \mu \mathrm{m}$ band of 0.36 . This plateau was introduced to better fit the extracted VSG component of Rapacioli et al. (2005) and Berné et al. (2007). The continuum model is described in the following section.

\subsection{The continuum}

In Rapacioli et al. (2005) and Berné et al. (2007) it was shown that some continuum emission is spatially associated with the band emission of eVSGs. In mild UV field PDRs, the continuum emission is observed to be linear up to $\sim 20 \mu \mathrm{m}$ (Berné et al. 2007; Compiègne et al. 2008). In high UV field PDRs, such as the Orion Bar, the shape of the continuum is observed to change due to a contribution from big grains at thermal equilibrium. For most of our sources we found that the 5.5-14 $\mu$ m continuum can be fitted by a single slope. For high UV field PDRs a bilinear continuum is used. The choice of the continuum (single slope or bilinear) can be set by the user in PAHTAT.

\subsection{Minor PAH features}

Three additional features are present in the mid-IR spectra of most PDRs, at 6.7, 11.55 and $13.5 \mu \mathrm{m}$. These features are best represented by three lorentzians at $6.7,11.55$ and $13.5 \mu \mathrm{m}$ with a $F W H M$ of $0.2,0.4$ and $0.25 \mu \mathrm{m}$, respectively. These features also appear in the $\mathrm{PAH}^{+}$and $\mathrm{PAH}^{0}$ components of Rapacioli et al. (2005). However, they are much fainter than the principal PAH features and were not included in the band template spectra. Their intensities are left as free parameters in the fit. However, these features are minor and their introduction does not change the results significantly.

\subsection{Gas lines}

Several gas lines fall in the 5.5-14 $\mu \mathrm{m}$ range, such as the $\mathrm{H}_{2} \mathrm{~S}(2)$ to $S(5)$ rotational lines, and fine structure lines from ionised gas such as [ArII], [NeII] and [SIV]. Some of these lines may be blended with PAH/eVSG features at the spectral resolution of ISOCAM-cvf and IRS-SL. Therefore, these features cannot be simply subtracted but they rather need to be treated simultaneously with the PAH and eVSG bands. In PAHTAT, we assumed Gaussian profiles with a fixed central wavelength and a FWHM given by the average spectral resolution $R$ of the instrument $(R=45$ for ISOCAM, and $R=80$ for IRS).

\subsection{Extinction correction}

Finally, our model takes into account extinction along the line of sight by dust within the PDR, which is caused mainly by silicate 


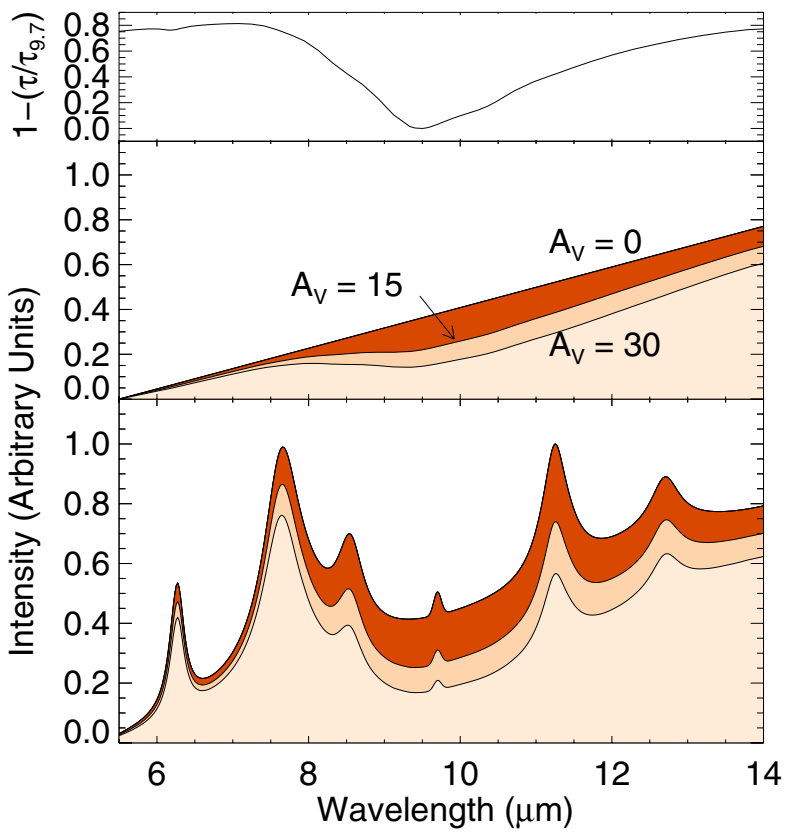

Fig. 2. Effect of the extinction correction on a linear continuum and on a synthetic PDR spectrum composed of continuum, band emission, and gas lines.

grains. Assuming that the emitting and absorbing materials in the considered column of material are fully mixed ${ }^{3}$ (see, for instance Disney et al. 1989; Smith et al. 2007b) leads to a correction factor $\left(1-\mathrm{e}^{-\tau_{\lambda}}\right) / \tau_{\lambda}$. The optical depth $\tau_{\lambda}$ at each wavelength is calculated using the relation

$\tau_{\lambda}=C_{\text {ext }}(\lambda) \times N_{\mathrm{H}}$,

where $C_{\text {ext }}(\lambda)$ is the extinction cross-section per $\mathrm{H}$ nucleon, calculated by Weingartner \& Draine (2001) for $R_{\mathrm{V}}=5.5$.

\subsection{Summary of the model}

In summary, the modelled mid-IR emission $I_{\text {model }}$ is written as

$I_{\text {model }}=\left(I_{\text {bands }}+I_{\text {continuum }}+I_{\text {gas }}+I_{\text {mbands }}\right) \frac{1 \mathrm{e}^{-C_{\text {ext }} N_{\mathrm{H}}}}{C_{\text {ext }} N_{\mathrm{H}}}$.

The adjusted parameters are $I_{\text {bands }}$, which represents the intensity of the band template spectra of PAH $\mathrm{H}^{0,+, x}$ and $\mathrm{eVSGs}, I_{\text {continuum, }}$ which is the (bi-)linear continuum emission, $I_{\text {gas }}$, which consists of the entire mid-IR $\mathrm{H}_{2}$ and ionised gas lines, and $I_{\text {mbands }}$ to account for the minor band features at 6.7, 11.5 and $13.5 \mu \mathrm{m}$. The hydrogen column density $N_{\mathrm{H}}$ is left as a free parameter in the fit. Hereafter, $N_{\mathrm{H}}$ will be expressed, for simplicity, in magnitudes of visual extinction along the line of sight, defined as $A_{\mathrm{V}}=N_{\mathrm{H}} /\left(1.8 \times 10^{21} \mathrm{~cm}^{-2}\right) \mathrm{mag}$. We tested the effect of using different extinction curves, corresponding to different values of $R_{\mathrm{V}}$, and found a difference of up to $20 \%$ in the estimated column density but no significant effect on the population weights when using $R_{\mathrm{V}}=3.1$ instead of $R_{\mathrm{V}}=5.5$. The extinction correction can significantly modify the shape and intensity of the mid-IR spectrum. As an example, Fig. 2 shows the effect of the extinction correction to a linear continuum and a synthetic spectrum for different values of $A_{\mathrm{V}}$. This shows an example of how

\footnotetext{
${ }_{3}$ PAHTAT gives the possibility to also use a foreground extinction correction $\mathrm{e}^{-\tau \lambda}$, although we assumed a mixed extinction model here.
}

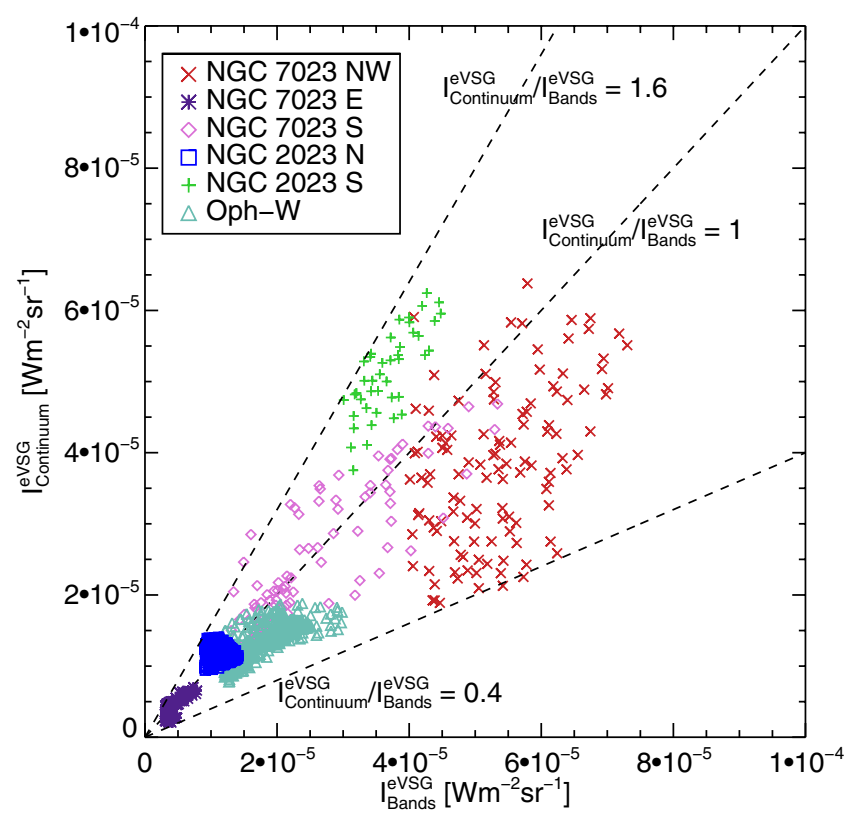

Fig. 3. Pixel-to-pixel correlation of the two components $I_{\text {bands }}^{\text {eVSG }}$ and $I_{\text {continum }}^{\text {evS }}$ for the resolved PDRs of Table 1. For each PDR, a mask was applied to show only the brightest pixels in $I_{\mathrm{eVSG}}^{\mathrm{bands}}$, i.e. the pixels towards the PDR front (see also Fig. 10).

different (i.e. non-linear) continua can be obtained using just a linear continuum and an extinction correction. On the other hand, to obtain significant information on the column density, this must be high enough to modify the spectrum significantly. This happens at an $A_{\mathrm{V}} \gtrsim 3$, which we defined as our threshold.

\section{7. $I_{0}$, the emission radiated by AIB carriers}

The total emission radiated by AIB carriers in both aromatic bands and mid-IR continuum of eVSGs (cf. Sect. 3.2) is given by

$I_{0}=I_{\mathrm{bands}}+I_{\text {continuum }}^{\mathrm{eVSG}}=I_{\mathrm{bands}}^{\mathrm{PAH}}+I_{\mathrm{bands}}^{\mathrm{eVSG}}+I_{\text {continuum }}^{\mathrm{eVSG}}$,

where $I_{\text {bands }}^{\mathrm{PAH}}$ is the intensity of all the $\mathrm{PAH}$ populations $\left(\mathrm{PAH}^{+}\right.$, $\mathrm{PAH}^{0}$ and $\left.\mathrm{PAH}^{x}\right) . I_{\text {bands }}^{\mathrm{eVSG}}$ and $I_{\text {continuum }}^{\mathrm{eVSG}}$ are the contribution of eVSGs to the bands and the continuum, respectively. While $I_{\text {bands }}^{\text {eVSG }}$ is a direct output of PAHTAT, $I_{\text {continuum }}^{\text {eVSG }}$ has to be computed separately. Considering that in moderate UV field regions $\left(G_{0} \lesssim 4000\right)$ emission from big grains is negligible at $\lambda \lesssim 14 \mu \mathrm{m}$, we considered that for these PDRs $I_{\text {continuum }}^{\mathrm{eVSG}}=I_{\text {continuum. Hence }}$ using the data in this region, we can calibrate a relation between $I_{\text {continuum }}^{\text {eVS }}$ and $I_{\text {bands }}^{\text {eVSG }}$. Figure 3 shows the pixel-to-pixel correlation of the two extracted components $I_{\mathrm{bang}}^{\mathrm{eVS}}$ and $I_{\text {continuum }}$ for all resolved PDRs (where $G_{0} \lesssim 4000$ ). The dispersion of this correlation suggests that the band and continuum emissions are not strictly related and that the band-to-continuum ratio can be affected by factors that still have to be understood, such as chemical composition and size distribution. To quantify the continuum intensity we therefore used the limits given by the cloud of points in Fig. 3, which gives $I_{\text {continuum }}^{\mathrm{eVSG}}=(1 \pm 0.6) \times I_{\text {bands }}^{\mathrm{eVSG}}$. The contribution of eVSGs to emission at longer wavelengths $(\lambda \gtrsim 14 \mu \mathrm{m})$ is neglected based on the results by Draine \& Li (2007), who showed that it is not significant for PAH grains of size $\lesssim 10 \AA$ ( $\sim 1200 \mathrm{C}$ atoms), which includes the size of eVSGs (Rapacioli et al. 2005). 
P. Pilleri et al.: Evaporating very small grains as tracers of the UV field in PDRs

Table 2. Spitzer IRS observational parameters and the assumed physical parameters for PDRs with mixed mid-IR populations.

\begin{tabular}{lccccc}
\hline \hline Object & $\begin{array}{c}\text { Position } \\
{\left[\alpha_{2000}, \delta_{2000}\right]}\end{array}$ & Aperture & $G_{0}$ & $\begin{array}{c}n_{\mathrm{H}} \\
{\left[\mathrm{cm}^{-3}\right]}\end{array}$ & Ref. \\
\hline Horsehead & $(05: 40: 53.8 ;-02: 28: 00)$ & $9^{\prime \prime} \times 7^{\prime \prime}$ & 100 & $2 \times 10^{5}$ & Habart et al. (2005) \\
Ced 201 & $(22: 13: 25 ; 70: 15: 03)$ & $22^{\prime \prime} \times 22^{\prime \prime}$ & 300 & $4 \times 10^{2}$ & Young Owl et al. (2002) \\
IC 63 & $(00: 59: 01 ; 60: 53: 19)$ & $30^{\prime \prime} \times 40^{\prime \prime}$ & 1100 & $1 \times 10^{5}$ & Gerin et al. (2003) \\
Parsamyan 18 S & $(06: 59: 41 ;-07: 46: 45)$ & $9^{\prime \prime} \times 8^{\prime \prime}$ & 3500 & $1 \times 10^{4}$ & Ryder et al. (1998) \\
Parsamyan 18 N & $(06: 59: 41 ;-07: 46: 12)$ & $4^{\prime \prime} \times 8^{\prime \prime}$ & 5000 & $1 \times 10^{4}$ & Ryder et al. (1998) \\
Orion Bar & $(05: 35: 21.4 ;-05: 25: 15)$ & $4^{\prime \prime} \times 8^{\prime \prime}$ & $4 \times 10^{4}$ & $2 \times 10^{5}$ & Tauber et al. (1994) \\
\hline
\end{tabular}

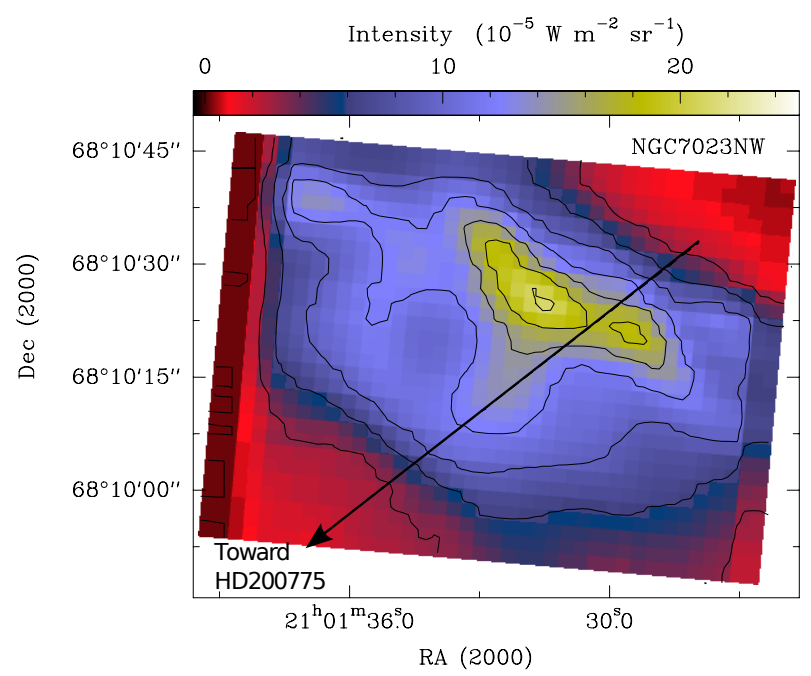

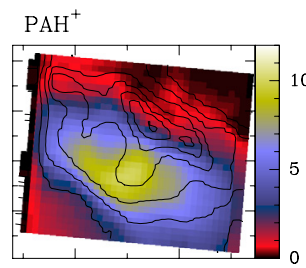
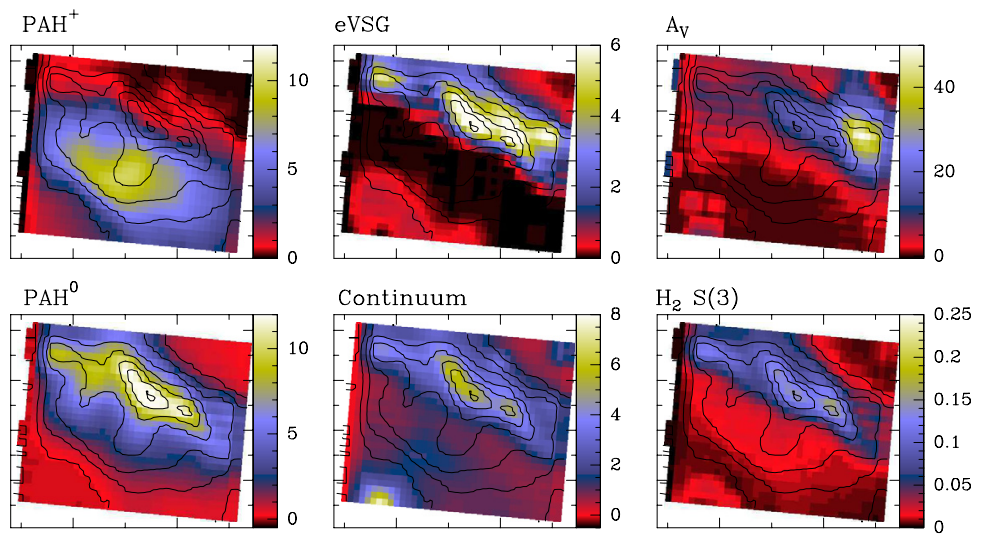

Fig. 4. Maps of the different components obtained using PAHTAT to fit the observed mid-IR spectra of the NGC 7023 NW IRS spectral cube. Integrated intensities in the 5.5-14 $\mu \mathrm{m}$ range of the total emission (left map) and of the different band template components (central panels) are displayed. The map of the column density expressed in $A_{\mathrm{V}}$ is also shown, as well as the flux in the $\mathrm{H}_{2} \mathrm{~S}(3)$ line (right maps). The black arrow

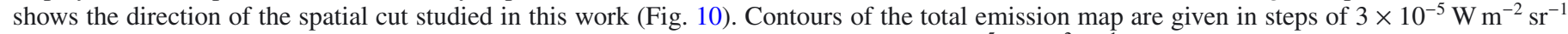
and are reported for reference in all the maps. The color intensity scales are in units of $10^{-5} \mathrm{~W} \mathrm{~m}^{-2} \mathrm{sr}^{-1}$, while the column density is expressed in magnitude of visual extinction. Results for the other resolved PDRs can be found in Fig. 9.

After defining the total eVSG emission, we can use in the following the ratio of this value to the total AIB emission as a tracer of the evolution of eVSGs in different environments:

$f_{\mathrm{eVSG}}=\frac{I_{\mathrm{bands}}^{\mathrm{eVSG}}+I_{\mathrm{continuum}}^{\mathrm{eVSG}}}{I_{0}}=(2 \pm 0.6) \times \frac{I_{\mathrm{bands}}^{\mathrm{eVSG}}}{I_{0}}$,

which can be derived directly from PAHTAT.

\section{Results of PAHTAT}

We used PAHTAT to fit the emission spectra of the PDRs with spatially resolved (Table 1) and mixed (Table 2) mid-IR populations.

\subsection{PDRs with spatially resolved mid-IR populations}

We applied PAHTAT for each spatial pixel of the spectroimagery cubes of the resolved PDRs listed in Table 1 . This yields the spatial distributions of the different emission components, as well as the column density in each pixel. Figure 4 shows the maps obtained for NGC 7023 NW. Maps for the other PDRs are displayed in Fig. 9. The spatial distributions of the $\mathrm{PAH}^{+}$, $\mathrm{PAH}^{0}$ and eVSG components are found to be close to those reported in previous studies (Rapacioli et al. 2005; Berné et al. 2007; Rosenberg et al. 2011). The comparison between the distributions of $A_{\mathrm{V}}$ and the AIB emission is consistent with the interpretation that the mid-IR emission is dominated by the excited edge of a dense structure (cloud or filament). The column densities determined with this method are in line with those derived from other tracers (see for example Habart et al. 2003; Gerin et al. 1998) and agree in absolute values within a factor of 4. The spatial patterns of the $A_{\mathrm{V}}$ maps seem indeed to be associated with variations in the physical or geometrical properties. For instance, the peak of column density observed in NGC $7023 \mathrm{NW}$ perfectly matches the high-density region traced by CS emission (Pilleri et al., in prep.).

\subsection{PDRs with mixed mid-IR populations}

Figure 5 shows the observed spectra of the PDRs with mixed mid-IR populations and their fits, whose main results are reported in Table 3 . The quality of the fit can be evaluated using the signal-to-noise ratio $(\mathrm{S} / \mathrm{R})$, defined as the ratio between the power of the fit divided by the power of the residuals (see Table 3). All S/Rs are above $100(20 \mathrm{~dB})$, which attests to the good quality of the fit. The extinction we derive is compatible with previous estimates that used molecular tracers or (sub-)millimeter dust emission (see, for example, Tauber et al. 1994; Kemper et al. 1999; Habart et al. 2005; Karr et al. 2005). The weights of the different populations vary in the PDRs, reflecting a difference in chemical abundances. $\mathrm{PAH}^{x}$ were suggested to be present only in highly excited regions (Joblin et al. 2008; Berné et al. 2009a), and not in mild UV-field reflection 

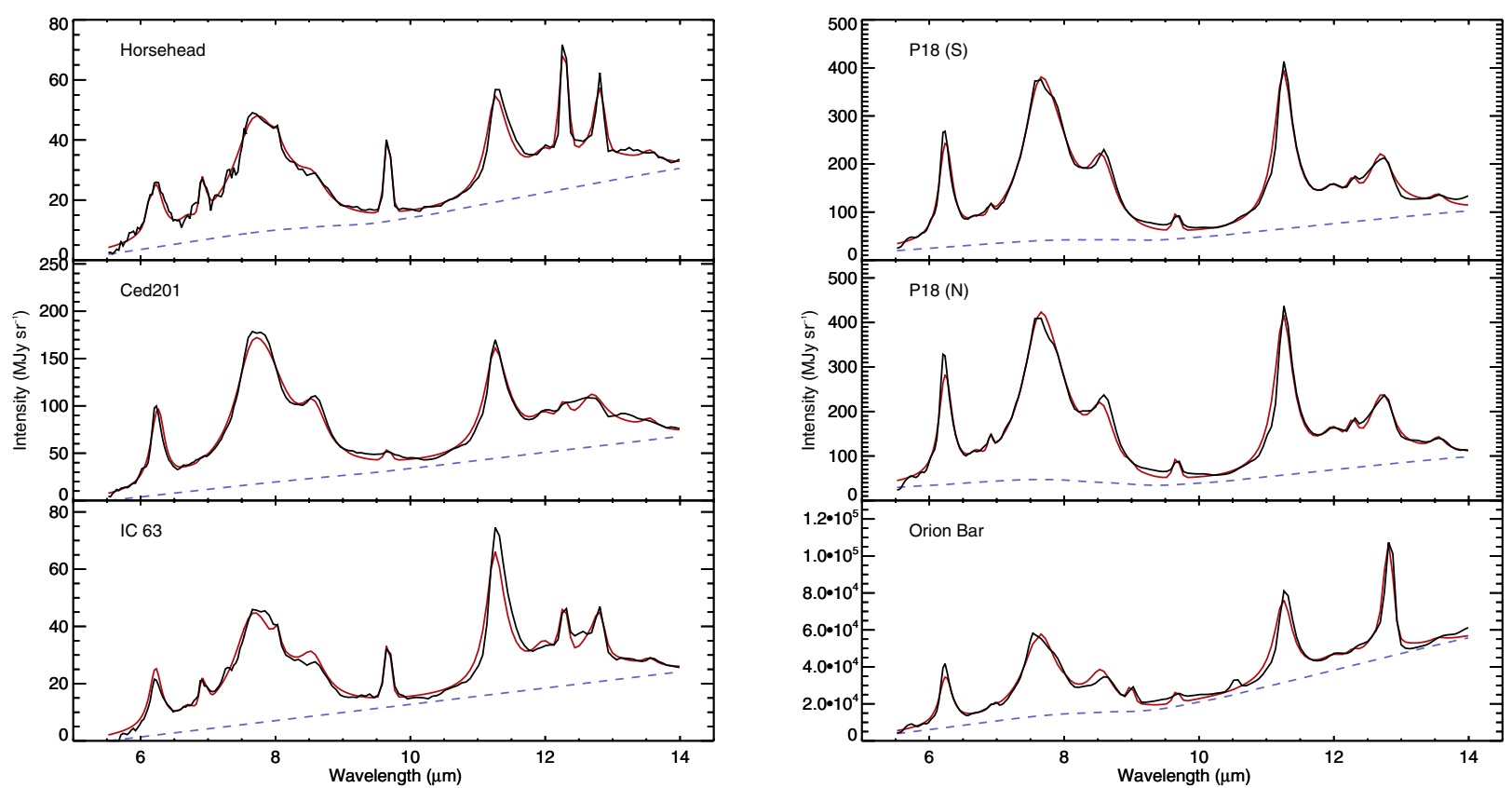

Fig. 5. Mid-IR spectra (black) obtained with IRS and their fit with PAHTAT (red) for the PDRs listed in Table 2. All quantities derived from the fit are reported in Table 3. The dashed line represents the continuum determined by the fit, corrected for the extinction along the line of sight.

Table 3. Results of PAHTAT for the PDR with mixed mid-IR populations.

\begin{tabular}{|c|c|c|c|c|c|c|c|c|c|c|c|}
\hline \multirow[t]{2}{*}{ Source } & \multicolumn{4}{|c|}{ Integrated intensities $\left[\mathrm{W} \mathrm{m}^{-2} \mathrm{sr}^{-1}\right]$} & \multicolumn{4}{|c|}{ PAH and eVSG fractions [\% $]^{a}$} & \multicolumn{3}{|c|}{ Results } \\
\hline & $I_{\mathrm{obs}}$ & $I_{\text {bands }}$ & $I_{\text {continuum }}$ & $I_{\text {gas }}$ & $\mathrm{PAH}^{0}$ & $\mathrm{PAH}^{+}$ & $\mathrm{PAH}^{x}$ & eVSG & $S / R^{b}$ & $f_{\mathrm{eVSG}^{c}}$ & $A_{\mathrm{V}}$ \\
\hline Horsehead & $8.3 \times 10^{-6}$ & $4.1 \times 10^{-6}$ & $3.7 \times 10^{-6}$ & $3.9 \times 10^{-7}$ & 32 & 2 & 1 & 64 & 238 & 0.78 & 6.5 \\
\hline Ced 201 & $2.4 \times 10^{-5}$ & $1.6 \times 10^{-5}$ & $7.6 \times 10^{-6}$ & $1.2 \times 10^{-7}$ & 21 & 21 & 11 & 45 & 281 & 0.63 & $\lesssim 3$ \\
\hline IC 63 & $7.2 \times 10^{-6}$ & $4.2 \times 10^{-6}$ & $2.8 \times 10^{-6}$ & $2.1 \times 10^{-7}$ & 54 & 0 & 0 & 45 & 111 & 0.63 & $\lesssim 3$ \\
\hline P18 S & $4.8 \times 10^{-5}$ & $3.2 \times 10^{-5}$ & $1.5 \times 10^{-6}$ & $3.5 \times 10^{-7}$ & 48 & 18 & 10 & 22 & 303 & 0.37 & $\tilde{10.0}$ \\
\hline P18 N & $5.1 \times 10^{-5}$ & $3.4 \times 10^{-5}$ & $1.5 \times 10^{-5}$ & $5.0 \times 10^{-7}$ & 57 & 13 & 11 & 17 & 173 & 0.30 & 21.2 \\
\hline Orion Bar & $9.9 \times 10^{-3}$ & $3.9 \times 10^{-3}$ & $5.8 \times 10^{-3}$ & $4.8 \times 10^{-5}$ & 59 & 32 & 0 & 8 & 161 & 0.15 & 8.9 \\
\hline
\end{tabular}

Notes. ${ }^{(a)}$ Relative to $I_{\text {bands }} ;{ }^{(b)} S / R=\int I_{\text {model }}^{2}(\lambda) / \int\left(I_{\text {model }}(\lambda)-I_{\text {obs }}(\lambda)\right)^{2} ;{ }^{(c)}$ fraction of C atoms locked in eVSGs. See Eq. (4) for details.

nebulae (Rapacioli et al. 2005; Berné et al. 2007). Indeed, $\mathrm{PAH}^{x}$ represent a minor fraction of the AIB emission $(\lesssim 10 \%)$ except for the nucleus of the starburst galaxy M 82 .

\section{Linking the chemistry with the physical conditions}

Rapacioli et al. (2005) and Berné et al. (2007) have suggested that eVSGs might be PAH clusters that evaporate into free-flying PAH molecules when exposed to UV radiation in PDRs. In the following, we assume that all carbon contained in eVSGs is transferred into PAHs, i.e. that the number of $\mathrm{C}$ atoms per unit volume contained in AIB carriers, $n_{\mathrm{C}}=n_{\mathrm{C}}^{\mathrm{eVSG}}+n_{\mathrm{C}}^{\mathrm{PAH}}$, is conserved. We have shown in Joblin et al. (2010) that there are two independent ways to derive the abundance of $\mathrm{C}$ in the mid-IR carriers, either by analysing the intensity of the mid-IR emission or by using the total column density derived from the fit. We have checked that the ratio of these two quantities varies by less than $10 \%$ in the cut of NGC7023 NW shown in Fig. 10, excluding strong variation in the abundance of carbon at the transition eVSG/PAH.

In Appendix A.1 we show that the emissivity per carbon atom of eVSGs and PAHs can be considered as similar. This means that $f_{\mathrm{eVSG}}$ directly represents the fraction of carbon atoms locked up in eVSGs compared to all carbon atoms in the AIB carriers. Therefore, we can quantify the evolution of the abundance of eVSGs as a function of the physical conditions $\left(G_{0}\right.$ and the gas density $\left.n_{\mathrm{H}}\right)$ by using the quantity $f_{\mathrm{eVsG}}$, which can be directly derived from the observations (cf. Eq. (4)).

\subsection{The relation between $G_{0}$ and $f_{\mathrm{eVsG}}$}

For each of the spatially resolved PDRs, we focused on one cut that extends perpendicularly from the star's position to the PDR and a few arcsecs past the PDR front (see Figs. 4 and 9). Qualitatively, the variation of $f_{\mathrm{eVSG}}$, calculated using Eq. (4), along each of the cuts shows that the eVSG abundance increases from the PDR front into the inner layers and reaches its maximum just behind the front. The carbon content in eVSGs decreases significantly in the more exposed part of the PDRs, confirming the photo-evaporation scenario.

To quantify this process, we need an estimate of the value of $G_{0}$ at various depths of the PDR along this cut, which is not known a priori. This was performed using the approach presented in Habart et al. (2003) for the Oph-W filament. This method relies on the modelling of the AIB emission using a few 
P. Pilleri et al.: Evaporating very small grains as tracers of the UV field in PDRs

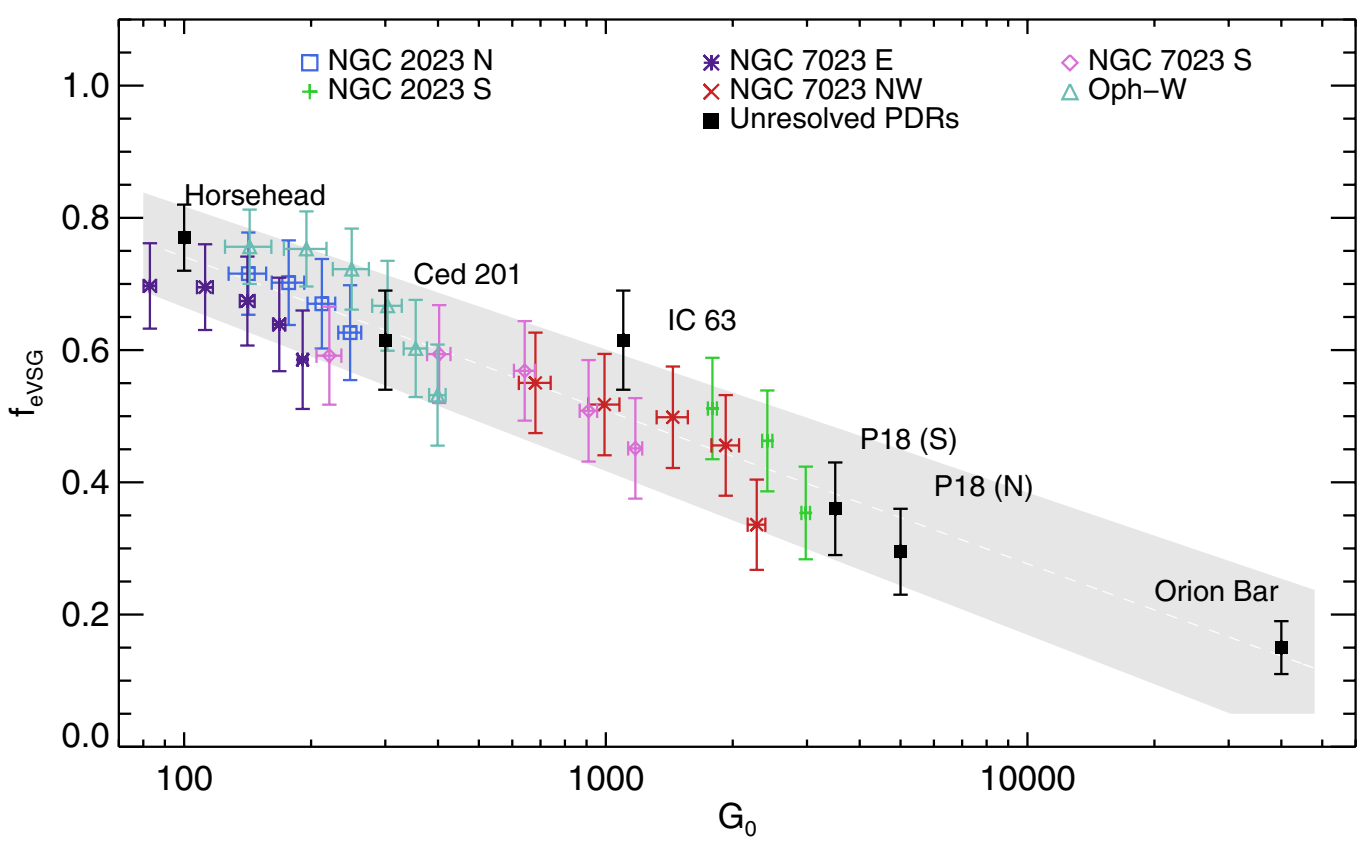

Fig. 6. Fraction of carbon atoms locked in eVSGs relative to the total carbon in the AIB carriers (derived with PAHTAT) as a function of the intensity of the local UV radiation field. The error bars result from the propagation of the uncertainty on the intensity of the eVSG continuum (see Sect. 3.7).

physical and geometrical assumptions ${ }^{4}$. This yields the values of $G_{0}$ and $n_{\mathrm{H}}$ at various depths in the PDR (upper panels in Fig. 10), which can be compared with the variation of $f_{\mathrm{eVSG}}$ (lowest panels in Fig. 10).

In Fig. 6, we report the values of $f_{\mathrm{eVSG}}$ as a function of the intensity of the UV radiation field $G_{0}$ for all regions. For each cut, the number of points that are reported depends on the spatial resolution of the observations. In each spatially resolved PDR, we excluded the low-density $\left(n_{\mathrm{H}} \lesssim 5 \times 10^{3} \mathrm{~cm}^{-3}\right)$ layers that lie in the most exposed region of the PDR (up to an $\left.A_{\mathrm{V}} \sim 0.1-0.3\right)$. In these regions, eVSGs are underabundant because the photoevaporation process is expected to be much faster than reformation. Hence their contribution to the midIR emission is minor and the derivation of their abundance is subject to large uncertainties. Instead, we concentrated on the PAH-eVSG transition, which arises at $A_{\mathrm{V}} \sim 0.5$ and is spatially coincident with the $\mathrm{H}_{2}$ pure rotational emission. This corresponds to regions in which fresh matter from dense regions is exposed to UV photons. Finally, we also added one point for each of the PDR with mixed mid-IR populations, for which we relied on the values of $G_{0}$ and $n_{\mathrm{H}}$ that are commonly used in the literature (Table 2).

Figure 6 shows a clear decrease of the fraction of carbon in eVSGs with increasing intensity of the UV radiation field. The trend can be fitted by the expression

$f_{\text {eVSG }}=(-0.23 \pm 0.02) \log _{10}\left(G_{0}\right)+(1.21 \pm 0.05)$.

This implies that $f_{\text {evSG }}$ can be used to trace the (local) intensity of the UV radiation field. At low UV field intensities $\left(G_{0} \lesssim 100\right)$, the mid-IR fits are less reliable because the AIB emission is fainter and sometimes barely detected, therefore the $f_{\text {evsG }}$ vs. $G_{0}$ relationship cannot be established. Similarly, the extrapolation of the relation between $f_{\mathrm{eVSG}}$ and $G_{0}$ to more intense UV fields

\footnotetext{
4 For the sake of completeness and to facilitate reading, the description of this method is reported in Appendix A.
}

(e.g. above $5 \times 10^{4}$, which is our limit in this paper) is also difficult because in these environments, the photo-processing of the eVSG and PAH populations is extreme and leads to the difficulty of observing the more fragile eVSG species, and likely breaks the assumption on the conservation of $n_{\mathrm{C}}$ in the AIB carriers. We conclude that our procedure to derive $G_{0}$ is reliable in the range $100 \lesssim G_{0} \lesssim 5 \times 10^{4}$. The gas densities that have been explored span the range $10^{2}-10^{5} \mathrm{~cm}^{-3}$.

\subsection{Correlation with other tracers}

Figure 7 displays the correlation between $f_{\text {evsG }}$ and the local density for the PDRs with both mixed and spatially resolved mid-IR populations, showing that these two quantities are uncorrelated. Similarly, no correlation appears between $f_{\mathrm{eVSG}}$ and the ratio $G_{0} / n_{\mathrm{H}}$, despite the link of this ratio with the fraction of ionised PAHs by Fleming et al. (2010) in their detailed study of NGC 7023 NW, NGC 2023 N, and NGC 2023 S. This proves that the evaporation of the eVSG population is indeed directly linked to the intensity of the UV radiation field through a mechanism that differs significantly from those driving the ionisation balance. This strongly suggests that the reformation of eVSGs is not efficient in these environments. Rapacioli et al. (2006) have indeed shown that the photo-evaporation of PAH clusters in the NGC 7023 NW PDR is much faster than their reformation by collisions with PAHs.

\subsection{Use of PAHTAT to derive $G_{0}$ or other parameters}

In principle, PAHTAT can be used to fit any mid-IR spectrum that shows AIB emission to provide $f_{\mathrm{eVSG}}$ and therefore the local value of $G_{0}$. However, when using this tool, one must bear in mind for which frame this relationship is applicable. PAHTAT was calibrated in relatively dense and highly UV-irradiated environments that are found in star-forming regions. This implies 1 ) that the exciting and evaporating radiation field results 


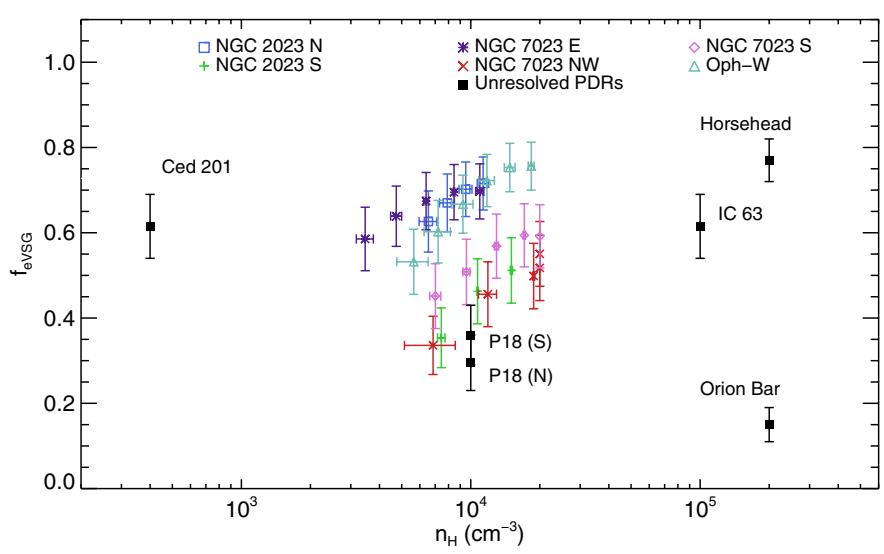

Fig. 7. Relation between the local density and the $f_{\text {eVSG }}$ value for the PDRs with spatially resolved ( Table 1) and mixed (Table 2) mid-IR populations. Error bars are calculated as in Fig. 6.

from massive stars so that the radiation field is dominated by UV photons; and 2) the presence of dense regions where eVSGs are abundant and start to evaporate where the UV irradiation penetrates. In other types of regions, the value of $G_{0}$ derived from PAHTAT may not be reliable. Still, PAHTAT can be used to identify unusual spectral properties of AIBs as related to peculiar properties of the source. Vega et al. (2010) for instance used the PAHTAT templates and identified an exceptionally intense $11.3 \mu \mathrm{m}$ features in early-type galaxies. They have interpreted this as a population of large neutral PAHs, which are not included in our templates.

The mid-IR emission of star-forming galaxies mainly arises from unresolved dense and highly UV-irradiated PDRs in which PAHTAT could be used to derive an average radiation field. To test the reliability of the value of $G_{0}$ derived with PAHTAT on unresolved PDRs, we used the fitting tool to determine a value of $f_{\mathrm{eVSG}}$ for the average spectra calculated over more extended fields of view (up to 1 arcmin) in our template object, NGC $7023 \mathrm{NW}$. This can be compared with $\left\langle f_{\mathrm{eVSG}}\right\rangle$, the mean value obtained by averaging results on the individual pixels contained in the same region. The two values agree well for regions close to the eVSG peak. For regions close to the PDR front, the value of $\left\langle f_{\mathrm{eVSG}}\right\rangle$ is higher but by less than 0.05 . This yields an overestimate of the mean UV radiation field by less than $50 \%$. Although this error is not directly applicable to other PDRs, it shows that the method can be used to derive an effective UV radiation field intensity in unresolved sources within a factor $\sim 2$.

As an example, we applied our fitting procedure to the IRS spectra of the nucleus and outflow of the starburst galaxy M 82. The fit results are shown in Fig. 8. The M 82 IRS spectral map has been studied previously by Beirão et al. (2008). They found that the magnitudes of visual extinction were ranging between $A_{\mathrm{V}}=0$ to 50 and were likely responsible for most of the spectral variations in the AIB features. The $A_{\mathrm{V}}$ values derived from our decomposition on each pixel are consistent with these values. The results of the fit applied to the mean spectra of the nucleus and the outflow of M 82 are still consistent with the results from Beirão et al. (2008), because we derive an estimate of the extinction along the line of sight of $A_{\mathrm{V}} \sim 21$ for the nucleus and $\sim 11$ for the outflow. The values of $f_{\mathrm{eVSG}}$ derived from the mid-IR fit correspond to a UV radiation field of $G_{0}=3_{-1}^{+2} \times 10^{3}$ for the nucleus and $G_{0}=(2 \pm 1) \times 10^{3}$ for the outflow. Previous studies using chemical modelling of the nucleus of M 82 by Fuente et al. (2008) have predicted a

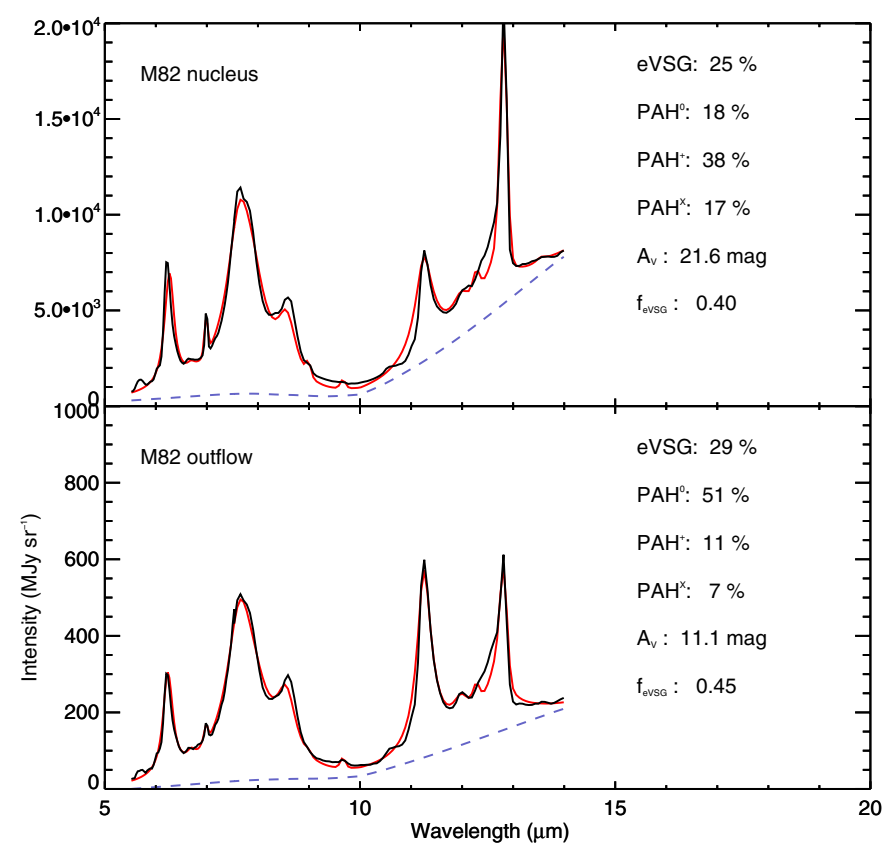

Fig. 8. Mid-IR spectra (black line) of the nucleus and the outflow of M 82 and their fits (red line) obtained with PAHTAT. The results of the fit suggest that the UV radiation field in the nucleus is slightly higher, as well as the column density of the gas.

value of $G_{0} \sim 1 \times 10^{4}$, whereas those using far-IR observations (Colbert et al. 1999; Kaufman et al. 1999) report lower values $\left(10^{2.8} \lesssim G_{0} \lesssim 10^{3.5}\right)$. These agree well with our estimates.

\section{Conclusion}

We have presented a new method for analysing the mid-IR spectra of PDRs using a basis of PAH and eVSG template spectra. We have shown that with a few, physically meaningful parameters it is possible to obtain a very good fit of the mid-IR spectra of PDRs that span a wide range of physical conditions. The fit returns the relative contribution of the PAH and eVSG populations to the mid-IR emission, the intensity of the gas lines (especially the rotational lines of $\mathrm{H}_{2}$ ), and the magnitudes of visual dust extinction along the line of sight.

We derived the abundance of $\mathrm{C}$ atoms locked in eVSGs relative to the total abundance of carbon in the AIB carriers. This value strongly correlates with the local UV field intensity and can therefore be used to quantify this important physical parameter. Our study has provided new insights into the properties of eVSGs, supporting a chemical scenario in which these species are destroyed by UV photons to produce free-flying PAHs. PAH clusters and PAH-Fe complexes have been proposed as plausible carriers for these eVSGs and detailed studies on the survival of these species in PDRs have been performed (Rapacioli et al. 2006; Simon \& Joblin 2009). One important conclusion of our observational study is that eVSGs are destroyed in PDRs faster than they can be reformed. This suggests that they are formed in the denser parts of molecular clouds, although this scenario will be difficult to probe since in these regions mid-IR emission cannot be triggered and therefore eVSG fingerprints cannot be observed. Another intriguing aspect is the continuum emission that seems to be associated to eVSGs. We showed that the intensity of this continuum is quite variable relative to the band intensity, likely reflecting a chemical or size distribution effect. Solving 
P. Pilleri et al.: Evaporating very small grains as tracers of the UV field in PDRs
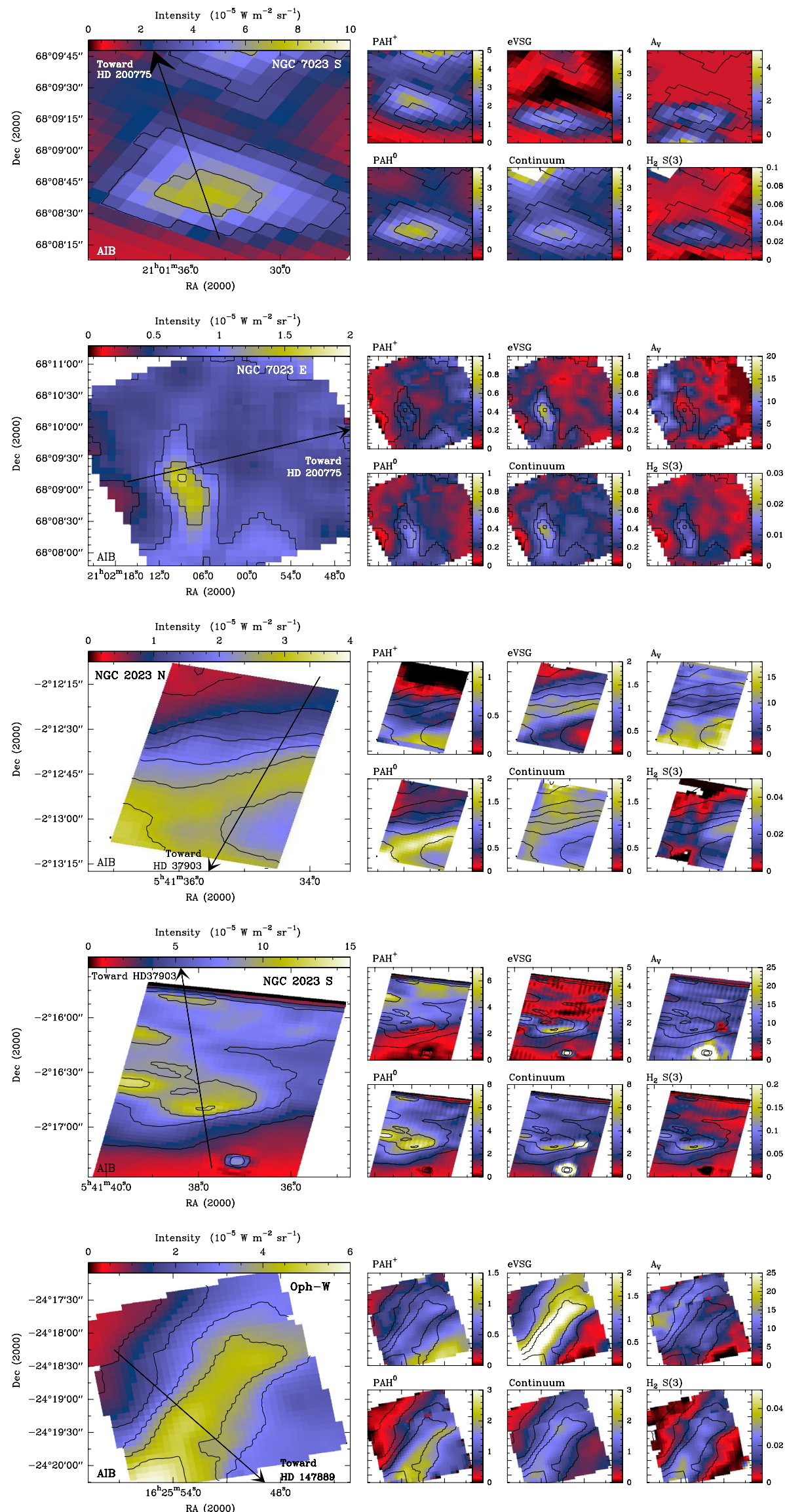

Fig. 9. Same as Fig. 4 but for the other sources considered in this work: from top to bottom, NGC 7023 S, NGC 7023 E, NGC 2023 N, NGC 2023 S, and the $\rho$-Ophiucus Filament. The solid lines represent the cuts studied in this work, whose analysis is shown in Fig. 10. 

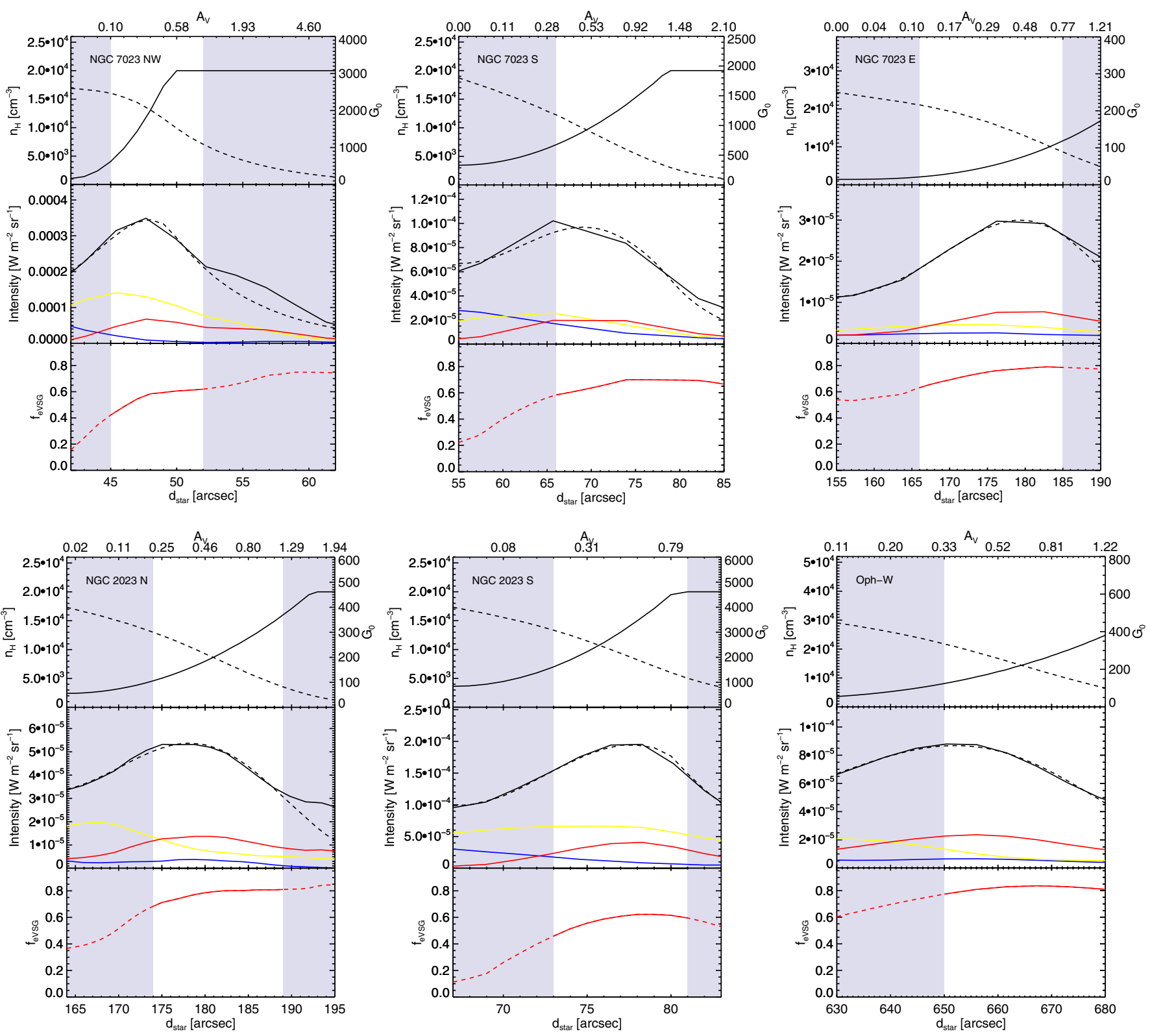

Fig. 10. Compilation of our results along each PDR cut defined in Figs. 4 and 9. Upper panels: the local density and UV radiation field profiles as a function of the distance from the star along the $r$ or $A_{\mathrm{V}}$ axis of Fig. A.1. Central panels: blue, yellow, and red represent the contribution of $\mathrm{PAH}^{+}, \mathrm{PAH}^{0}$ and eVSGs, respectively, as extracted from the fit. Solid black lines represent the corrected mid-IR emission profile. The dotted lines represent the fit obtained with the geometrical model described in Appendix A. Lower panels: the variation in the fraction of carbon atoms locked in eVSGs relative to the total carbon in all the AIB carriers. The grey-shaded regions are those that were not considered in Fig. 6 and Eq. (5).

this question opens new perspectives for studies on laboratory analogues of eVSGs.

Finally, our fitting code PAHTAT (PAH Toulouse Astronomical Templates) is made publicly available to the community as an IDL package, together with the template spectra used in this work. This procedure combined with the high spatial resolution and sensitivity of forthcoming missions such as JWST and SPICA will allow us to determine the spatial variation of the UV radiation field in more distant galaxies and protoplanetary disks.

Acknowledgements. We thank the referees for useful comments that improved the clarity of the paper. This work was supported by the French National Program, Physique et Chimie du Milieu Interstellaire, which is gratefully acknowledged. This paper was also partially supported by the Spanish program CONSOLIDER INGENIO 2010, under grant CSD2009-00038 Molecular Astrophysics: The Herschel and ALMA Era (ASTROMOL).

\section{Appendix A: Mid-IR emission model}

In this section, we describe how we derived the local physical parameters, i.e. the gas density $\left(n_{\mathrm{H}}\right)$ and the UV radiation field $\left(G_{0}\right)$ from the local mid-IR emission of the AIB carriers.

\section{A.1. Energy balance}

The UV absorption cross-section of PAHs was measured to be proportional to the number of involved carbons (Joblin et al. 1992). This has also been shown on a larger sample of species using theoretical calculations ${ }^{5}$ (Malloci et al. 2004, 2007). Using a typical value for $\sigma_{\mathrm{C}}^{\mathrm{PAH}}$, one can estimate that screening atoms inside a grain by surface atoms is negligible for grains with

5 http://astrochemistry.ca.astro.it/database/ 
lesser than $\sim 5 \times 10^{4}$ carbon atoms. As mentioned in Sect. 5, eVSGs considered in these work are relatively small, containing up to about 1200 carbon atoms $(\sim 10 \AA)$, and we therefore considered that the absorption cross sections per $\mathrm{C}$ atom of eVSGs and PAHs are similar: $\sigma_{\mathrm{C}}^{\text {eVSG }}=\sigma_{\mathrm{C}}^{\mathrm{PAH}}=\sigma_{\mathrm{C}}$.

The power absorbed by the AIB carriers can be expressed as

$P_{\mathrm{abs}}=\int_{91.2 \mathrm{~nm}}^{\lambda_{\max }} n_{\mathrm{C}} \sigma_{\mathrm{C}}(\lambda) F(\lambda) \mathrm{d} \lambda$,

where $F(\lambda)$ is the flux of the radiation field from the nearby star. Since ionised PAHs and large neutral PAHs (and hence eVSGs) can absorb significantly in the visible (Salama et al. 1996), we assume $\lambda_{\max }=1000 \mathrm{~nm}$.

As discussed by different authors (Boulanger \& Perault 1988; Habart et al. 2003), the power emitted by the AIB carriers can be expressed as a function of $G_{0}$ :

$P_{\mathrm{em}}=\epsilon \times n_{\mathrm{H}} \times G_{0}$,

where $G_{0}$ denotes the UV radiation field in Habing units. Considering that all species absorb with the same $\sigma_{\mathrm{C}}$ and applying the conservation of energy, an emissivity per $C$ atom of the AIB carriers can be defined as $\epsilon_{\mathrm{C}}^{\mathrm{eVSG}}=\epsilon_{\mathrm{C}}^{\mathrm{PAH}}=\epsilon_{\mathrm{C}}$. The emissivity $\epsilon$ can be conveniently expressed as

$\epsilon=n_{\mathrm{C}} \epsilon_{\mathrm{C}} / n_{\mathrm{H}}$

where $n_{\mathrm{C}}$ is the number of $\mathrm{C}$ atoms per cubic centimeter that is locked up in the AIB carriers. Writing the energy balance $P_{\mathrm{em}}=$ $P_{\text {abs }}$ leads to

$\epsilon=n_{\mathrm{C}} / n_{\mathrm{H}} \frac{1}{G_{0}} \times \int_{91.2 \mathrm{~nm}}^{1000 \mathrm{~nm}} \sigma_{\mathrm{C}}(\lambda) F(\lambda) \mathrm{d} \lambda$.

We computed $\epsilon$ for a variety of PAHs of different sizes and charges using the values of $\sigma_{\mathrm{C}}$ available from the on-line database of Malloci et al. (2007). The results scatter within $10 \%$. Assuming a value of $n_{\mathrm{C}} / n_{\mathrm{H}}=4.2 \times 10^{-5}$ (Draine 2003, for $R_{\mathrm{V}}=5.5$ ) we obtain $\epsilon \sim 5 \times 10^{-32} \mathrm{~W} \mathrm{H}^{-1}$, consistent with previous estimates by Habart et al. (2001) and Habart et al. (2003). Equation (A.3) shows that the value of $\epsilon$ depends a priori on the spectral shape (hardness) of the radiation field. To estimate the variation of $\epsilon$ with the hardness of the radiation field, we calculated it with the appropriate Kurucz spectra for the stars of Table 1. Our calculations show that for the objects studied in this paper, $\epsilon$ marginally depends on the hardness of the radiation field.

\section{A.2. Determining of the structure of the PDRs with spatially resolved mid-IR populations}

The concepts presented in the previous section can be used to study the physical conditions in PDRs with spatially resolved mid-IR populations (Table 1). However, to directly relate the observations to the local UV radiation field and gas density, some knowledge on the geometry of the PDR is needed. We assumed a spherical shell model as in Habart et al. (2003, see also Fig. A.1), in which the cloud is divided into successive layers of increasing density. The star is placed at the centre of the cavity, at a distance $d_{\text {front }}$ that is determined by the projected distance on the sky (cf. Table 1). The total intensity radiated by AIB carriers as a function of the position on the sky is obtained by integrating Eq. (A.2) along a given line of sight, i.e.:

$I(z)=\frac{1}{4 \pi} \int_{0}^{L} \epsilon \times n_{\mathrm{H}}(r) \times G_{0}(r) \mathrm{d} l$,

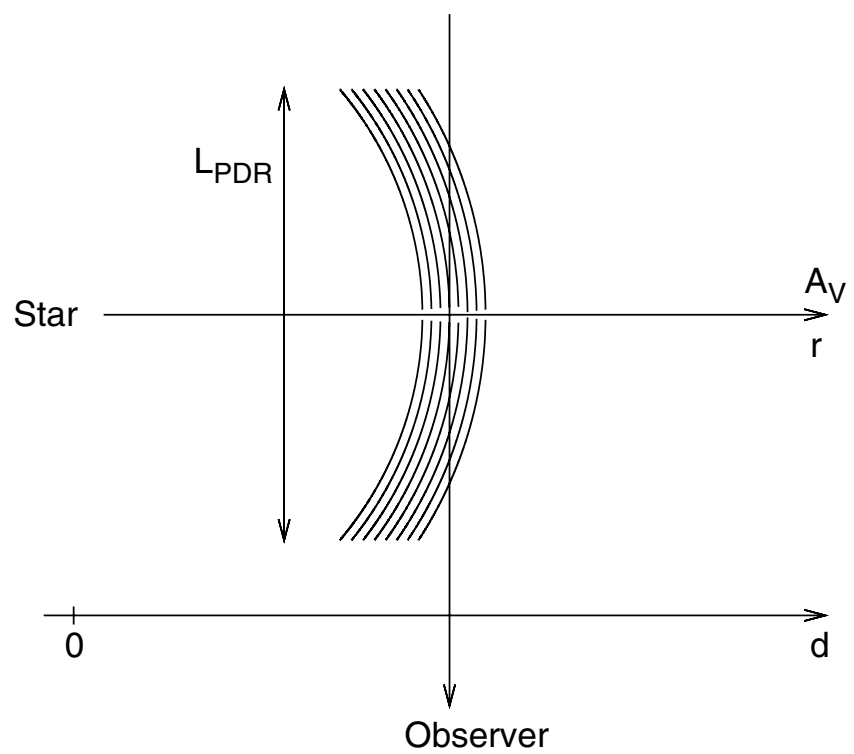

Fig. A.1. Spherical shell geometry used to model the PDRs with spatially resolved mid-IR populations (Table 1). The $r$ and $A_{\mathrm{V}}$ axis refers to the local position inside the PDR, along the star-PDR axis, counted from the star and from the PDR front, respectively. The $d$ axis refers to the position of the line of sight on the sky, counted from the star position.

where $z$ is the projected distance from the front, $r$ is the distance from the star, and $L$ is the thickness of the PDR perpendicularly to the plane of the sky. The local radiation field inside the PDR depends on the impinging radiation field at the PDR surface and on the gas density profile that determines its attenuation. It evolves as

$G_{0}(r)=G_{0}^{\text {front }} \times \mathrm{e}^{-\sigma_{\mathrm{UV}} \int_{0}^{r} n_{\mathrm{H}}(r) \mathrm{d} r}$,

where $\sigma_{\mathrm{UV}}$ is the dust extinction cross-section at $1000 \AA$ (1.5 $\times$ $10^{-21} \mathrm{~cm}^{2} \mathrm{H}^{-1}$, Weingartner \& Draine 2001). The density profile is modelled between the front position at $r=d_{\text {front }}$ and a cutoff position $r=d_{\text {front }}+d_{0}$ as a power law from the density $n_{\mathrm{H}}^{0}$ in the diffuse region close to the star, up to a maximum density $n_{\mathrm{H}}^{\text {plateau }}$. Beyond the cutoff position, the density is assumed to be constant, equal to $n_{\mathrm{H}}^{\text {plateau }}$ :

$$
\begin{aligned}
n_{\mathrm{H}}(r) & =n_{\mathrm{H}}^{0}+n_{\mathrm{H}}^{\text {plateau }} \times\left[\frac{r-\mathrm{d}_{\text {front }}}{\mathrm{d}_{0}}\right]^{\alpha} & \text { if } d_{\text {front }}<r<d_{0} \\
& =n_{\mathrm{H}}^{\text {plateau }} & \text { if } r>d_{0},
\end{aligned}
$$

where the gas density in the plateau $\left(n_{\mathrm{H}}^{\text {plateau }}\right)$ was determined by molecular observations (cf. references in Table A.1) and $d_{\text {front }}$ was evaluated at half of the peak intensity of the mid-IR emission (Figs. 4 and 9). $G_{0}^{\text {front }}$ has been evaluated as explained in Sect. 2. The free parameters of the fit are $n_{\mathrm{H}}^{0}, \alpha$ and $d_{0}$, which define the gas density profile, and the cloud physical length $L$. The fit results for each one of the PDRs with spatially resolved mid-IR populations are summarised in Table A.1, and the modelled profiles are reported in the middle panels of Fig. 10. Along with $n_{\mathrm{H}}^{0}, \alpha$ and $d_{0}$, the model enables one to calculate the values of the UV radiation field $G_{0}$ along the cuts (Fig. 10). Good fits to $I_{0}$ are obtained (Fig. 10), except for the deepest parts of the PDRs, mainly at $d \gtrsim d_{0}$ where the density is poorly constrained. 
Table A.1. Parameters for the gas density profiles in the PDRs with spatially resolved mid-IR populations, extracted from our emission model as explained in Appendix A.2.

\begin{tabular}{lcccccc}
\hline \hline Object & $\begin{array}{c}d_{\text {front }} \\
{[\mathrm{pc}]}\end{array}$ & $\begin{array}{c}n_{\mathrm{H}}^{\text {plateau }} \\
{\left[\mathrm{cm}^{-3}\right]}\end{array}$ & $\begin{array}{c}n_{\mathrm{H}}^{0} \\
{\left[10^{3} \mathrm{~cm}^{-3}\right]}\end{array}$ & $\alpha$ & $d_{0}$ & $L$ \\
& {$[\mathrm{pc}]$} & {$[\mathrm{pc}]$} \\
\hline NGC 7023 NW & 0.087 & $2 \times 10^{4 a}$ & 1.1 & 2.0 & 0.016 & 0.13 \\
NGC 7023 S & 0.11 & $2 \times 10^{4 a}$ & 3.4 & 2.0 & 0.054 & 0.10 \\
NGC 7023 E & 0.32 & $3 \times 10^{4 a}$ & 1.4 & 2.7 & 0.091 & 0.27 \\
NGC 2023 N & 0.38 & $2 \times 10^{4 b}$ & 2.4 & 2.0 & 0.069 & 0.27 \\
NGC 2023 S & 0.13 & $2 \times 10^{4 b}$ & 3.6 & 2.0 & 0.033 & 0.07 \\
Oph-W & 0.40 & $4 \times 10^{4 c}$ & 2.6 & 2.5 & 0.051 & 0.28 \\
\hline
\end{tabular}

References. ${ }^{(a)}$ Gerin et al. (1998); ${ }^{(b)}$ Fuente et al. (1995); ${ }^{(c)}$ Habart et al. (2003).

Since we concentrated our analysis around the AIB peak, this discrepancy does not affect the rest of our analysis.

\section{References}

Alecian, E., Catala, C., Wade, G. A., et al. 2008, MNRAS, 385, 391

Allamandola, L. J., Tielens, A. G. G. M., \& Barker, J. R. 1985, ApJ, 290, L25

Beirão, P., Brandl, B. R., Appleton, P. N., et al. 2008, ApJ, 676, 304

Berné, O., Joblin, C., Deville, Y., et al. 2007, A\&A, 469, 575

Berné, O., Fuente, A., Goicoechea, J. R., et al. 2009a, ApJ, 706, L160

Berné, O., Joblin, C., Fuente, A., \& Ménard, F. 2009b, A\&A, 495, 827

Boulanger, F., \& Perault, M. 1988, ApJ, 330, 964

Boulanger, F., Lorente, R., Miville Deschênes, M. A., et al. 2005, A\&A, 436, 1151

Burton, M. G., Howe, J. E., Geballe, T. R., \& Brand, P. W. J. L. 1998, PASA, 15, 194

Cesarsky, D., Lequeux, J., Ryter, C., \& Gérin, M. 2000, A\&A, 354, L87

Chokshi, A., Tielens, A. G. G. M., Werner, M. W., \& Castelaz, M. W. 1988, ApJ, 334,803

Colbert, J. W., Malkan, M. A., Clegg, P. E., et al. 1999, ApJ, 511, 721

Compiègne, M., Abergel, A., Verstraete, L., \& Habart, E. 2008, A\&A, 491, 797

Désert, F. X., Boulanger, F., Léger, A., Puget, J. L., \& Sellgren, K. 1986, A\&A, 159,328

Désert, F., Boulanger, F., \& Puget, J. L. 1990, A\&A, 237, 215

Diplas, A., \& Savage, B. D. 1994, ApJS, 93, 211

Disney, M., Davies, J., \& Phillipps, S. 1989, MNRAS, 239, 939

Draine, B. T. 2003, ARA\&A, 41, 241

Draine, B. T., \& Li, A. 2007, ApJ, 657, 810

Finkenzeller, U. 1985, A\&A, 151, 340

Fleming, B., France, K., Lupu, R. E., \& McCandliss, S. R. 2010, ApJ, 725, 159

Fuente, A., Martin-Pintado, J., \& Gaume, R. 1995, ApJ, 442, L33

Fuente, A., Martín-Pintado, J., Rodríguez-Fernández, N. J., et al. 1999, ApJ, 518, L45

Fuente, A., García-Burillo, S., Usero, A., et al. 2008, A\&A, 492, 675

Gerin, M., Phillips, T. G., Keene, J., Betz, A. L., \& Boreiko, R. T. 1998, ApJ, 500,329

Gerin, M., Fossé, D., \& Roueff, E. 2003, in SFChem 2002: Chemistry as a Diagnostic of Star Formation, ed. C. L. Curry, \& M. Fich, 81

Habart, E., Verstraete, L., Boulanger, F., et al. 2001, A\&A, 373, 702
Habart, E., Boulanger, F., Verstraete, L., et al. 2003, A\&A, 397, 623

Habart, E., Abergel, A., Walmsley, C. M., Teyssier, D., \& Pety, J. 2005, A\&A, 437, 177

Habing, H. J. 1968, Bull. Astron. Inst. Netherlands, 19, 421

Houck, J. R., Roellig, T. L., van Cleve, J., et al. 2004, ApJS, 154, 18

Joblin, C., \& Tielens, A. G. G. M. 2011, PAHs and the Universe: A Symposium to Celebrate the 25th Anniversary of the PAH Hypothesis, EAS Publ. Ser., 46 Joblin, C., Léger, A., \& Martin, P. 1992, ApJ, 393, L79

Joblin, C., Szczerba, R., Berné, O., \& Szyszka, C. 2008, A\&A, 490, 189

Joblin, C., Pilleri, P., Montillaud, J., et al. 2010, A\&A, 521, L25

Karr, J. L., Noriega-Crespo, A., \& Martin, P. G. 2005, AJ, 129, 954

Kaufman, M. J., Wolfire, M. G., Hollenbach, D. J., \& Luhman, M. L. 1999, ApJ, 527, 795

Kemper, C., Spaans, M., Jansen, D. J., et al. 1999, ApJ, 515, 649

Kurucz, R. L. 1993, VizieR Online Data Catalog, 6039

Léger, A., \& Puget, J. L. 1984, A\&A, 137, L5

Li, A., \& Draine, B. T. 2001a, ApJ, 554, 778

Li, A., \& Draine, B. T. 2001b, ApJ, 550, L213

Malloci, G., Mulas, G., \& Joblin, C. 2004, A\&A, 426, 105

Malloci, G., Joblin, C., \& Mulas, G. 2007, Chem. Phys., 332, 353

Racine, R. 1968, AJ, 73, 233

Rapacioli, M., Joblin, C., \& Boissel, P. 2005, A\&A, 429, 193

Rapacioli, M., Calvo, F., Joblin, C., et al. 2006, A\&A, 460, 519

Rosenberg, M. J. F., Berné, O., Boersma, C., Allamandola, L. J., \& Tielens, A. G. G. M. 2011, A\&A, 532, A128

Ryder, S. D., Allen, L. E., Burton, M. G., Ashley, M. C. B., \& Storey, J. W. V. 1998, MNRAS, 294, 338

Salama, F., Bakes, E. L. O., Allamandola, L. J., \& Tielens, A. G. G. M. 1996, ApJ, 458, 621

Simon, A., \& Joblin, C. 2009, J. Phys. Chem. A, 113, 4878

Smith, J. D. T., Armus, L., Dale, D. A., et al. 2007a, PASP, 119, 1133

Smith, J. D. T., Draine, B. T., Dale, D. A., et al. 2007b, ApJ, 656, 770

Tauber, J. A., Tielens, A. G. G. M., Meixner, M., \& Goldsmith, P. F. 1994, ApJ, 422,136

van den Ancker, M. E., The, P. S., Tjin A Djie, H. R. E., et al. 1997, A\&A, 324, L33

Vega, O., Bressan, A., Panuzzo, P., et al. 2010, ApJ, 721, 1090

Weingartner, J. C., \& Draine, B. T. 2001, ApJ, 548, 296

Werner, M. W., Uchida, K. I., Sellgren, K., et al. 2004, ApJS, 154, 309

Witt, A. N., Gordon, K. D., Vijh, U. P., et al. 2006, ApJ, 636, 303

Young Owl, R. C., Meixner, M. M., Fong, D., et al. 2002, ApJ, 578, 885 